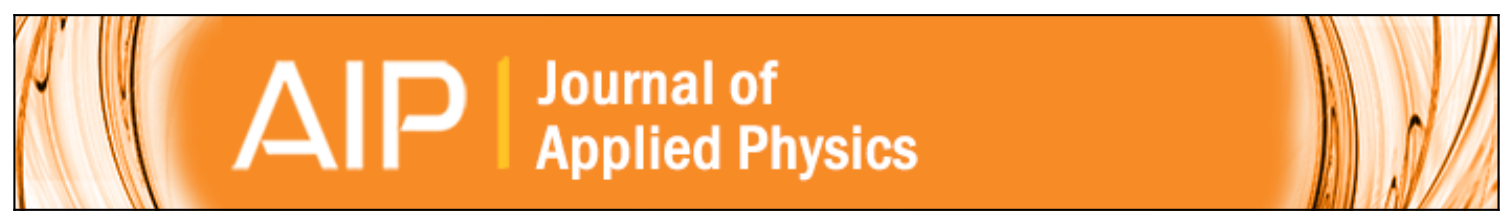

\title{
A simplified procedure for obtaining relative x-ray intensities when a texture and atomic displacements are present
}

Baoping He, Satish Rao, and C. R. Houska

Citation: Journal of Applied Physics 75, 4456 (1994); doi: 10.1063/1.355960

View online: http://dx.doi.org/10.1063/1.355960

View Table of Contents: http://scitation.aip.org/content/aip/journal/jap/75/9?ver=pdfcov

Published by the AIP Publishing

\section{Articles you may be interested in}

Measurements of relative $x$-ray line intensities and their application to a single standard procedure for quantitative $\mathrm{x}$-ray microanalysis

J. Appl. Phys. 100, 124312 (2006); 10.1063/1.2400089

Determination of Relative Intensity in X-Ray Reflection Study

J. Appl. Phys. 36, 3432 (1965); 10.1063/1.1703011

A Scintillation Counter for the Relative Determination of X-Ray Intensities in Radiological Work

Rev. Sci. Instrum. 22, 646 (1951); 10.1063/1.1746024

Analysis of Intensities Obtainable Using Pinhole and Slit Collimators in X-Ray Powder Diffraction Techniques

J. Appl. Phys. 21, 779 (1950); 10.1063/1.1699757

A Technique for Obtaining the Integrated Intensity from X-Ray Powder Photographs

Rev. Sci. Instrum. 9, 79 (1938); 10.1063/1.1752440

\section{MIT LINCOLN}

LABORATORY CAREERS

Discover the satisfaction of innovation and service

to the nation
- Space Control

- Air \& Missile Defense

- Communications Systems

\& Cyber Security

- Intelligence, Surveillance and

Reconnaissance Systems

\section{LINCOLN LABORATORY}

MASSACHUSETtS INSTITUTE OF TeCHNOLOGY

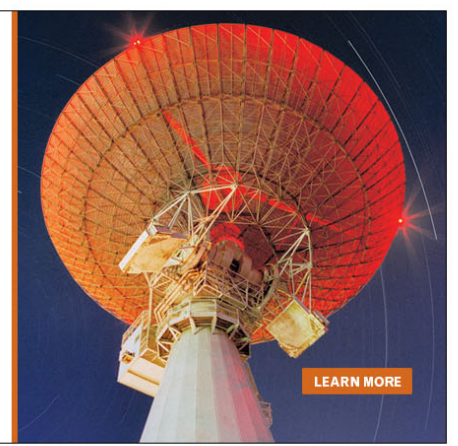




\title{
A simplified procedure for obtaining relative $x$-ray intensities when a texture and atomic displacements are present
}

\author{
Baoping He, Satish Rao, ${ }^{\text {a) }}$ and C. R. Houska \\ Department of Materials Engineering, Virginia Polytechnic Institute and State University, Blacksburg, \\ Virginia 24061
}

(Received 10 May 1993; accepted for publication 21 November 1993)

\begin{abstract}
The orientation problem in polycrystalline cubic materials has been simplified, using fundamental relationships, so that the determination of a quantitative interrelationship between the various Bragg peak intensities is no longer a formidable task. This is demonstrated with a cubic $\mathrm{Cu}-\mathrm{Be}-\mathrm{Co}$ alloy having a fiber texture, and a conventional focusing diffractometer. Because data are required which extend over a larger range in $d$ spacings, extinction, thermal, and static atomic displacements must be included into the analysis of intensities. The displacement terms and the extinction parameters may be of primary interest or used as a correction. Seventeen diffraction peaks are used in the example. These must be internally consistent with a crystallite orientation function, the cubic symmetry of the sample, extinction effects influencing the two strongest peaks, and attenuation due to atomic displacements. Tabulated coefficients are presented which greatly reduce the task of calculating the orientation function. A correction is given for instrumental smearing which should be considered for stronger textures than the intermediate case examined or for intermediate textures and nonfocusing instrumental conditions.
\end{abstract}

\section{INTRODUCTION}

Quantitative x-ray diffraction studies are typically carried out on either ideal powders or single crystals. Textured materials are often avoided because either assumptions must be made that weaken the final results or one must deal with a quantitative determination of the crystallite orientation function. Knowing the latter, would allow one to generate all measurements related to texture. The determination of the orientation function can be as time consuming as the determination of other structural parameters that may in fact be of primary interest. These might include: the percent transformation, and other parameters that effect the $x$-ray intensities such as: subtle changes in the positioning of atoms in the unit cell, displacement fields from lattice defects, and thermal atomic vibrations. Some of these effects can have a relatively small effect on the $x$-ray intensities and are most easily examined either in the nearly ideal powder form or as a single crystals. However, in limiting x-ray examinations to these extreme samples, one loses an understanding of the structure of most real crystalline materials that are of commercial importance. There is a need to develop an X-ray technique that is simple to use and one which can be applied directly to intensity data collected with a conventional parafocusing diffractometer. Although this instrumentation is not conventionally used for texture studies, it is often required to obtain the resolution for many secondary structural parameters obtained from x-ray diffraction.

We describe a simplified procedure for quantitative fiber texture analysis as it applies to data collection using a conventional parafocusing diffractometer. This requires a more complete analytical description of those factors that influence integrated $x$-ray intensities than what is being used in the

a) Now with Wright Laboratories, WPAFB, OH 45433. traditional pole figure approach. However, the latter form of data collection is ideal for those using $\mathrm{x}$-ray diffraction as a means to obtain the orientation function with the primary goal of calculating the average physical properties of a polycrystalline aggregate with a texture and secondary parameters are not needed.

In using conventional parafocusing diffractometers, the number of Bragg peaks sampled having different crystallographic orientations is limited in number and depends upon the radiation used. When the Bragg intensities are plotted in reciprocal space, they range from high intensity points near the origin to those at much larger distances. Extinction effects can very significantly reduce the intensities of the strongest intensities, while those higher order peaks with a small $d$ spacing can be significantly reduced by thermal vibrations and static displacements originating from atomic size differences. The latter effect can be particularly dominant for those alloys having large atomic size differences and undergoing the early stages of precipitation. When sample preparation involves annealing, subgrains can become sufficiently large to make an extinction correction essential, if the strongest peaks are to be interrelated statistically with the weaker peaks in a unified analysis. Consequently, static and thermal displacements, and extinction effects are included whereas these effects are traditionally ignored in a conventional texture analysis.

There is a considerable volume of literature already available dealing with pure texture analysis which had its quantitative origin with the works of Roe and Krigbaum, ${ }^{1}$ and Bunge. ${ }^{2}$ The latter provides a systematic review and contains the basic equations for those pursuing a conventional texture analysis with the goal of using the orientation function primarily for the understanding the physical properties found in polycrystalline samples. However, secondary factors not dealing with texture are not considered. This work 
begins with Refs. 1 and 3 and includes the previously mentioned correction terms.

In a quantitative intensity analysis, an artificial fiber texture may be introduced by spinning a sample about its normal. However, many specimens already have a fiber texture parallel to the surface normal. Under these conditions, the texture can be of major interest and be determined by methods given in this paper. In either case, the orientation function allows one to calculate the average scattering from a sample with a fiber texture. This is illustrated in a second paper which deals with temperature diffuse scattering. Similarly, the short range order diffuse scattering could also be calculated quantitatively from a textured sample using the procedure described along with parameters obtained from single crystal data.

Our approach is illustrated using an annealed $\mathrm{Cu}-\mathrm{Be}-\mathrm{Co}$ (Brush-Wellman 25 alloy). For completeness, instrumental smearing effects are also considered in connection with texture analysis. These can be of importance when the texture is relatively strong even with high resolution diffractometers. Consequently, this is included in the present analysis, and is considered when interrelating mathematically generated pole figures to the measured pole figure data. Instrumental smearing could introduce a limitation in the examination of strongly textured materials using conventional nonfocusing pole figure devices.

\section{INTEGRATED INTENSITY}

The integrated intensity from a polycrystalline material using a diffractometer equipped with a monochromater, is given by ${ }^{4-6}$

$$
P_{i}=k K_{i}(2 \theta) E_{i} g_{i} e^{-2\left(M_{T}+M_{S}\right)}
$$

with

$$
K_{i}(2 \theta)=\frac{I_{0} A_{0} \lambda^{3} w h}{\mu V_{c}^{2}} \frac{1+p \cos ^{2} 2 \theta}{(1+p) \sin ^{2} \theta \cos \theta} j F_{i}^{2}
$$

and $k=$ constant, $I_{0}=$ intensity of incident beam, $A_{0}=$ cross-sectional area of incident beam at the specimen, $\lambda=\mathrm{x}$-ray wavelength, $w$ and $h$ are the receiver slit width and height, $\mu=$ overall linear absorption coefficient of the alloy, $V_{c}=$ volume of an average unit cell in the matrix, $j=$ multiplicity factor of the normal Bragg peak, $F_{i}=$ structure factor, $g_{i}=$ orientation correction factor for planes $(h k l)=i\left(g_{i}=1\right.$ if the sample is an ideal powder), $p=$ the polarization factor which is one if no monochromator is used, $2 \theta=$ the diffraction angle for matrix scattering, and $M_{T}, M_{S}=$ thermal and static factors for the matrix. (If $p$ is not known from experiment, it is conventionally taken to be $\cos ^{2} 2 \theta^{\prime}$ where $2 \theta^{\prime}=$ diffraction angle from the monochromator. For a discussion of this term, and $K_{i}(2 \theta)$ see p. 544 of Ref. 4). $E_{i}$ represents an extinction correction which may be found in a simplified form in Ref. 7, pp. 111-118. Both $E_{i}$ and $g_{i}$ are introduced to modify the classical powder expression found in the literature. If primary extinction dominates secondary, $E_{i}=q$ with

$$
\begin{aligned}
q= & \frac{Q^{\prime}}{Q_{h k l}}=\tanh \left[2 t\left(\frac{Q \cot \theta}{\lambda\left(1+\cos ^{2} 2 \theta\right)}\right)^{1 / 2}\right] / \\
& 2 t\left(\frac{Q \cot \theta}{\lambda\left(1+\cos ^{2} 2 \theta\right)}\right)^{1 / 2}
\end{aligned}
$$

where $t$ is the thickness of a perfect plate-like crystal, $Q^{\prime}$ is the reflecting power from the dynamic theory, and $Q_{i}$ is from the kinematic theory or

$$
Q_{i}=r_{e}^{2}\left(\frac{1+\cos ^{2} 2 \theta}{2 \sin 2 \theta}\right)\left(\frac{\lambda^{3}}{V_{c}^{2}}\right) F_{i}^{2},
$$

where $r_{e}^{2}=7.94 \times 10^{26}\left(\mathrm{~cm}^{2}\right)$ is a constant. For secondary extinction, the sample absorption is corrected according to ${ }^{7}$

$$
E_{i}=\left[\frac{\mu}{\left(\mu+g q Q_{i}\right)}\right] q Q_{i} .
$$

From Eq. (2a), one finds that primary extinction introduces the correction $q(0<q<1)$ and from Eq. (2c) one finds a second parameter $g$ included with $\mu$, the linear absorption coefficient. These equations are based upon a mosaic block model with $g(0<g<1)$ depending upon the degree of a parallel alignment of subgrains within the grains. As $t$ increases the range of internal perfection within a grain becomes greater while a larger $g$ value indicates greater parallel alignment of subgrains. Taking $g=0$ would eliminate the effect of secondary extinction and $E_{i}=q$. These parameters are of interest in showing trends in crystalline perfection.

$M_{T}$ is commonly calculated from Debye theory. ${ }^{5} M_{S}$ will be treated in a later paper in some detail for systems in the early stages of aging or precipitation where $M_{S}$ can greatly dominate the corresponding thermal term, $M_{T}$. For the present, it is assumed that the system under consideration

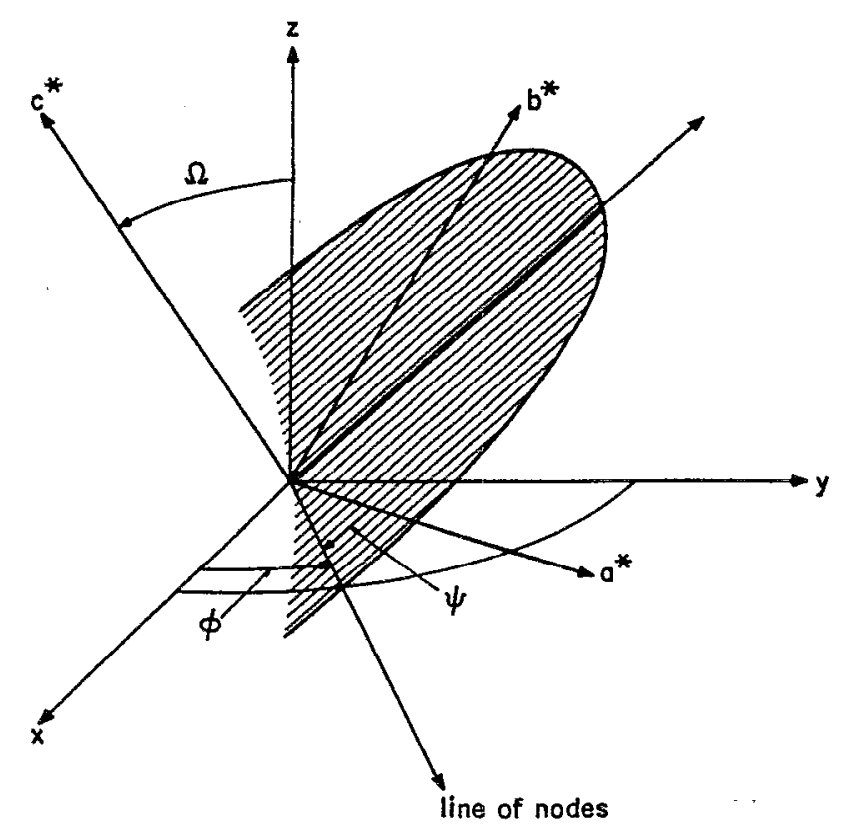

FIG. 1. Illustration of Euler angles $\Omega, \phi$ and $\psi$ as they relate to the reciprocal lattice of one crystallite. $Z$ is normal to the sample surface and parallel to the fiber axis. 
TABLE I. Coefficients $C_{h k l}^{n m}\left(C_{i j}\right)$ in terms of $(h k l)$ and $\chi$ angle to an order of $n=16$.

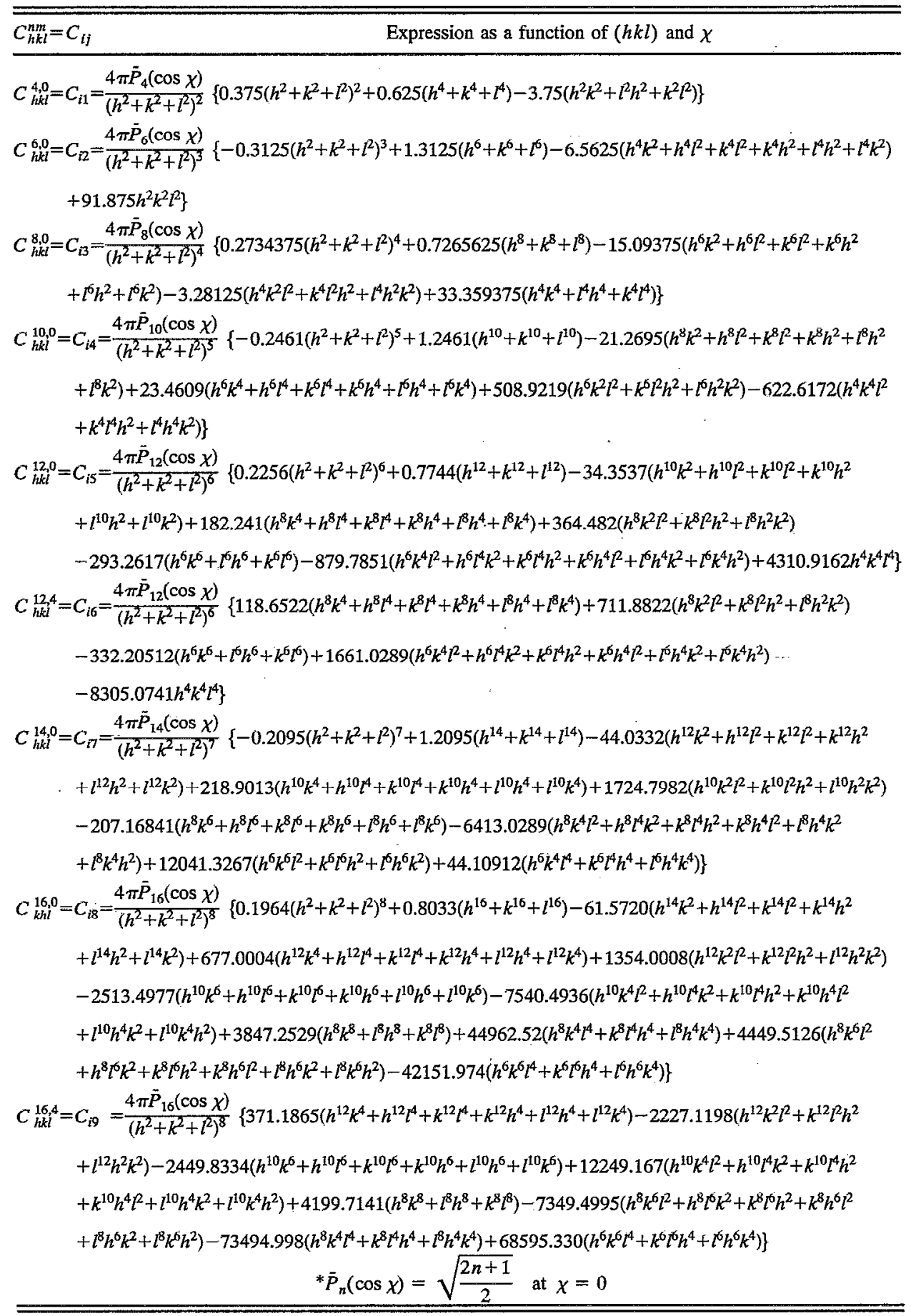

is a substitutional solid solution without severe static distortions introduced by an aging treatment. ${ }^{6}$ With this in mind, $2 M_{S}$ is given by

$$
2 M_{S}=D_{1} X_{B}\left(1-X_{B}\right)\left(\frac{1+\nu}{1-\nu}\right)^{2} V_{S F}^{2}\left(h^{2}+k^{2}+l^{2}\right)
$$

with $D_{1}=0.0587$ for $f c c$ and 0.0932 for $b c c$ lattices. Here $X_{B}$ is the atomic fraction of atom type $B, \nu=$ Poisson's ratio, $V_{S F}=(1 / v)\left(\partial_{v} / \partial X_{B}\right)$ is the volume size factor for $B$ atoms dissolved in an $A$ lattice, ${ }^{8}$ and $h k l$ are the Miller indices.
For the purpose of evaluating $g_{i}$, both sides of Eq. (1a) can be divided by $K_{i}(2 \theta) E_{i} e^{-2\left(M_{T}+M_{S}\right)}$, which will be defined as the reduced integrated intensity. This isolates $\left(k g_{i}\right)$ as a measurable result. Normally all quantities are known except $E_{i}$ which can be taken as unity for a first round of analysis. Also, if the slits and $\mathrm{x}$-ray tube conditions remain unchanged $I_{0} A_{0} \lambda^{3} w h$ can be taken as a constant and included in $k$.

For a sample with a fiber texture or an artificially generated fiber texture attained by sample rotation about the nor- 
mal, the texture is completely defined by the orientation function, $\omega(\Omega, \psi) . \omega(\Omega, \psi) \sin \Omega d \Omega d \psi$ is the probability of finding a crystallite oriented in the angular range between $(\Omega, \psi)$ and $(\Omega+d \Omega, \psi+d \psi) . \Omega, \psi$ and $\phi$ are the Euler angles shown in Fig. 1. In our case, the texture is independent of $\phi$ or is made independent by a continuous rotation of $\phi$. The orientation function is expressed as a series of spherical harmonics ${ }^{1}$

$$
\begin{aligned}
\omega(\Omega, \psi)= & \sum_{n=0}^{\infty} A_{n 0} \bar{P}_{n}^{0}(\cos \Omega) \\
& +2 \sum_{n=1}^{\infty} \sum_{m=0}^{n}\left[A_{n m} \bar{P}_{n}^{m}(\cos \Omega) \cos m \psi\right. \\
& \left.+B_{n m} \bar{P}_{n}^{m}(\cos \Omega) \sin m \psi\right],
\end{aligned}
$$

where $\bar{P}_{n}^{m}(\cos \Omega)$ is the normalized associated Legendre polynomial, $A_{n m}$ and $B_{n m}$ are coefficients determined from the intensity data. For an ideal powder, $\omega(\Omega, \psi)=\omega_{0}$, which is a constant independent of $\Omega$ and $\psi$. Taking the probability as 1 for all orientations, we have

$$
\omega_{0} \int_{0}^{2 \pi} \int_{0}^{\pi} \sin \Omega d \Omega d \psi=1, \quad \text { with } \omega_{0}=\frac{1}{4 \pi} .
$$

The function $\bar{\omega}(\Omega, \psi)$ gives the orientation distribution relative to the random or ideal powder

$$
\bar{\omega}(\Omega, \psi)=4 \pi \omega(\Omega, \psi) .
$$

The pole density function $g_{k k l}(\chi)$ can also be expanded as a series of Legendre polynomials,

$$
g_{h k l}(\chi)=\sum_{n=0}^{\infty} G_{n}^{h k l} \bar{P}_{n}(\cos \chi),
$$

where $\bar{P}_{n}(\cos \chi)$ are normalized Legendre polynomials. The coefficient $G_{n}^{h k l}$ is given by

$$
\begin{aligned}
G_{n}^{h k l}= & 4 \pi\left(\frac{2}{2 n+1}\right)^{1 / 2}\left\{A_{n 0} \bar{P}_{n}^{m}\left(\cos \Theta_{h k l}\right)\right. \\
& +2 \sum_{m=0}^{n}(-1)^{m}\left[A_{n m} \bar{P}_{n}^{m}\left(\cos \Theta_{h k l}\right) \cos m \Phi_{h k l}\right. \\
& \left.\left.+B_{n m} \bar{P}_{n}^{m}\left(\cos \Theta_{h k l}\right) \sin m \Phi_{h k l}\right]\right\}
\end{aligned}
$$

where $A_{n m}$ and $B_{n m}$ are the coefficients of the orientation function in Eq. (4). For a random powder all the coefficients vanish except $A_{0,0}=1 /(\sqrt{8 \pi})$, while for cubic crystallites, all the coefficients $B_{n m}$ vanish and $A_{n m} \neq 0$ only if $m$ is an integral multiple of four. Also, not all nonvanishing coefficients $A_{n m}$ are independent. The interdependency among the nonvanishing coefficients was given by $\operatorname{Roe}^{9}$ up to $n=22$ and by $\mathrm{RaO}^{3}$ up to $n=46$.

Equation (6a) can be greatly simplified using procedures discussed on pp. 1874-1877 of Ref. 3. These simplifications are originally presented in the Ph.D thesis by $\mathrm{Rao}^{10}$ and the

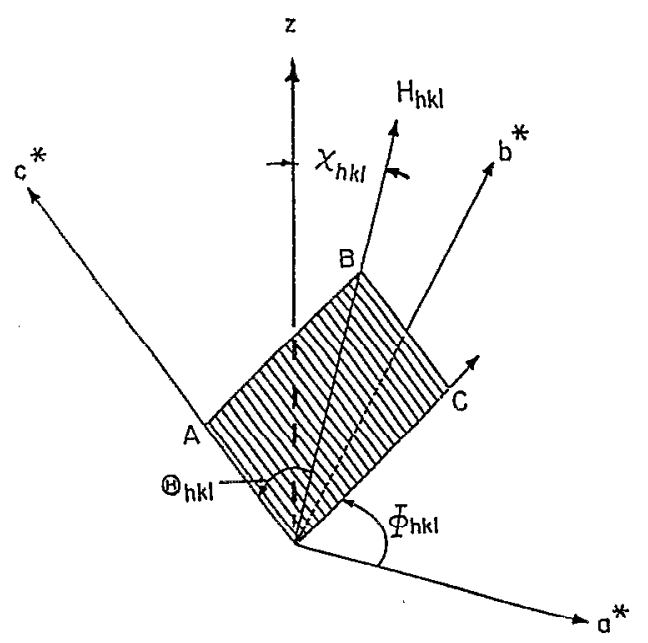

FIG. 2. Interrelationship of angles $\Theta_{h k l}$ (not the Bragg angle), $\Phi_{h k l}$, and $\chi$ to $H_{h k l}, a^{*}, b^{*}$, and $c^{*}$ in reciprocal lattice.

results tabulated on p. 250 in Appendix B. This has been put into a more convenient form for the present paper in Table I as a set of coefficients, $C_{i j}$, which enter into

$$
g_{h k l}=1+\sum_{n=1}^{N} \sum_{m=0}^{n} C_{h k t}^{n m} A_{n m}
$$

or simply

$$
g_{i}=1+\sum_{j=1}^{j_{0}} C_{i j} A_{j}(i=1,2, \ldots \ldots .,)
$$

Where $G_{0}^{h k l} \cdot \bar{P}_{0}(\cos \chi)=1, i$ designates different $(h k l)$ planes, $j$ different $n m$ combinations, and $j_{0}$ is the total number of the independent coefficients. The coefficients $C_{i j}$ are expressed as a function of $(h k l)$, and the $\chi$ angle to the 16th order in Table I.

When there is overlap of peaks at a $2 \theta$ posilion, the sum $I_{h k l}^{c}$ for all plane types should be combined and weighted according to their multiplicities $j_{1}$ and $j_{2}$. For example, when two types of planes $h_{1}, k_{1}, l_{1}$ and $h_{2}, k_{2}, l_{2}$ have identical $d$ spacings in a cubic crystal, the superposition equation is

$$
T_{12}=\frac{j_{1} T_{1}+j_{2} T_{2}}{p_{1}+p_{2}}
$$

where $T_{12}$ designates each of the following terms, $I_{h k l}^{c}, G_{n}^{h k l}$, $C_{h k l}^{n m}$, and $C_{i j}$ with the subscripts 1 and 2 standing for $h_{1}, k_{1}$, $l_{1}$ and $h_{2}, k_{2}, l_{2}$ respectively. (511) and (333), (600) and (442), and (731) and (553) overlap in the cubic system and are treated accordingly. 
TABLE II. Reduced $\left(k \cdot g_{h k l}\right)$ data for three Cu-Be samples.

\begin{tabular}{|c|c|c|c|c|c|}
\hline \multirow[b]{2}{*}{$h k l$} & \multicolumn{4}{|c|}{$\left(k \cdot g_{h k l}\right)$} & \multirow[b]{2}{*}{$\sigma_{-1}$} \\
\hline & A & B & $\mathrm{C}$ & Average & \\
\hline 111 & 1.000 & 1.000 & 1.000 & 1.000 & 0.000 \\
\hline 200 & 0.848 & 0.747 & 0.767 & 0.787 & 0.053 \\
\hline 220 & 0.128 & 0.150 & 0.140 & 0.139 & 0.011 \\
\hline 311 & 0.461 & 0.480 & 0.483 & 0.475 & 0.012 \\
\hline 222 & 1.382 & 1.231 & 1.424 & 1.346 & 0.102 \\
\hline 400 & 0.899 & 0.801 & 0.903 & 0.868 & 0.058 \\
\hline 331 & 0.356 & 0.338 & 0.358 & 0.351 & 0.011 \\
\hline 420 & 0.418 & 0.401 & 0.464 & 0.428 & 0.033 \\
\hline 422 & 0.643 & 0.653 & 0.724 & 0.673 & 0.044 \\
\hline $511+333$ & 0.694 & 0.709 & 0.861 & 0.755 & 0.092 \\
\hline 531 & 0.490 & 0.493 & 0.600 & 0.527 & 0.063 \\
\hline $600+442$ & 0.665 & 0.612 & 0.760 & 0.679 & 0.075 \\
\hline 620 & 0.501 & 0.518 & 0.580 & 0.533 & 0.042 \\
\hline 533 & 0.788 & 0.768 & 0.843 & 0.800 & 0.039 \\
\hline 622 & 0.420 & 0.507 & 0.535 & 0.487 & 0.060 \\
\hline 642 & 0.650 & 0.554 & 0.690 & 0.631 & 0.070 \\
\hline $731+553$ & 0.528 & 0.542 & 0.626 & 0.565 & 0.053 \\
\hline
\end{tabular}

In Eqs. (7a) and (7b), $g_{i}$ may be expressed as $(1 / k) \cdot\left(k g_{i}\right)$ since only $\left(k g_{i}\right)$ can be evaluated from the reduced experimental values of Eq. (1a) and $k$ is still to be determined. Rewriting Eq. (7b), we have

$$
\sum_{j=1}^{q} C_{i j} A_{j}-\frac{1}{k} \cdot\left(k g_{i}\right)=-1
$$

which allows the orientation function coefficients $A_{j}$ and the scaling factor $k$ to be obtained by a least squares fitting routine. To solve this linear system, at least $q+1$ independent $\left(k g_{i}\right)$ values at different orientations are required. The order of the orientation function is chosen using the maximum number of available peaks. Fewer peaks or terms are required for weak textures.

\section{A. Experimental example}

The fiber texture of a $\mathrm{Cu}-11.67$ at $\% \mathrm{Be}-0.23$ at $\% \mathrm{Co}$ alloy is used to illustrate the procedures described in the present work. Samples of $2.22 \mathrm{~cm}\left(7^{\prime \prime} / 8\right)$ diameter and 0.24 $\mathrm{cm}\left(3^{\prime \prime} / 32\right)$ thick were cut perpendicular to the axis of a hot extruded rod. This aligned the fiber axis to the surface normal. A solution treatment at $780^{\circ} \mathrm{C}$ for 15 min maintained the grain size at an upper level which gave reproducible intensity measurements by spinning in $\phi$ and rocking $X(=\omega)$ by $\pm 1.5^{\circ}$. Samples were rapidly quenched from the anneal- ing temperature into a $10 \% \mathrm{NaCl}$ water solution at room temperature and surface cleaned with a $15 \%$ nitric acid solution.

Integrated intensities were obtained using filtered $\mathrm{MoK}_{\alpha}$ radiation by scanning $2 \theta$ using a parafocusing diffractometer. The normalized data were reduced according to Eq. (1a) and are shown in Table II for three samples. In the first round of analysis, the (111) and (200) were excluded in favor of the equivalent orientations (222) and (400) which were essentially free from the effect of extinction.

The orientation function coefficients $A_{n m}$ and scaling factor $k$, calculated by linear least squares regression with Eq. (9) are listed in Table III using 6th to 16th order analyses. Pole densities calculated from $A_{n m}$ via Eq. (7b) are compared with measured data, scaled by $k$, in Table IV. The scaling factor $k$ calculated from different orders ranges from 0.572 to 0.583 . As expected, the agreement between the measured and calculated $g_{h k l}(0)$ from Eq. (7b) depends upon the order. Using the highest order available from the data gives the best agreement. The average difference for 16 th order is $2.6 \%$ with a maximum of $9.1 \%$ for (331). These are within experimental error, provided (111) and (200) are excluded or an extinction correction is included for these peaks.

Calculated $g_{h k l}$ values for the 16th order listed in Table IV show that the most favorable orientation is [111] with $g_{222}=2.32$ and the second favorable orientation is [100]

TABLE III. Scaling factor and orientation coefficient $A_{n m}$ for $n=6$ to $n=16$.

\begin{tabular}{|c|c|c|c|c|c|c|c|c|c|c|}
\hline$n$ & $k$ & $A_{4,0}$ & $A_{6,0}$ & $A_{8,0}$ & $A_{10,0}$ & $A_{12,0}$ & $A_{12,4}$ & $A_{14,0}$ & $A_{16,0}$ & $A_{16,4}$ \\
\hline 6 & 0.583 & -0.00581 & 0.01621 & & & & & & & \\
\hline 8 & 0.580 & -0.00656 & 0.01612 & 0.00402 & & & & & & \\
\hline 10 & 0.578 & -0.00521 & 0.01589 & 0.00433 & -0.00304 & & & & & \\
\hline 12 & 0.572 & -0.00737 & 0.01506 & 0.00295 & -0.00358 & 0.00594 & 0.00170 & & & \\
\hline 14 & 0.569 & -0.00845 & 0.01444 & 0.00218 & -0.00358 & 0.00654 & 0.00194 & 0.00170 & & \\
\hline 16 & 0.579 & -0.00497 & 0.01360 & 0.00307 & -0.00227 & 0.00659 & 0.00189 & 0.00169 & -0.00365 & 0.00049 \\
\hline
\end{tabular}


TABLE IV. The pole density $g_{h k l}$ calculated from $\left(k \cdot g_{h k l}\right)$ and Eq. $(9)$ for different orders.

\begin{tabular}{|c|c|c|c|c|c|c|c|}
\hline \multirow[b]{3}{*}{$h k l$} & \multicolumn{6}{|c|}{$g_{h k l}$ for different order } & \multirow[b]{3}{*}{16} \\
\hline & $\left(k \cdot g_{h k i}\right)$ & & & & & & \\
\hline & $k$ & 6 & 8 & 10 & 12 & 14 & \\
\hline 111 & 1.733 & 2.026 & 2.078 & 2.240 & 2.281 & 2.342 & 2.322 \\
\hline 200 & 1.364 & 1.364 & 1.489 & 1.405 & 1.512 & 1.543 & 1.511 \\
\hline 220 & 0.241 & 0.195 & 0.288 & 0.300 & 0.295 & 0.254 & 0.224 \\
\hline 311 & 0.823 & 1.035 & 0.946 & 0.896 & 0.842 & 0.891 & 0.828 \\
\hline 222 & 2.332 & 2.026 & 2.078 & 2.240 & 2.281 & 2.342 & 2.322 \\
\hline 400 & 1.504 & 1.364 & 1.489 & 1.405 & 1.512 & 1.543 & 1.511 \\
\hline 331 & 0.608 & 0.790 & 0.849 & 0.779 & 0.643 & 0.670 & 0.669 \\
\hline 420 & 0.741 & 0.616 & 0.582 & 0.716 & 0.846 & 0.855 & 0.752 \\
\hline 422 & 1.166 & 1.306 & 1.251 & 1.133 & 1.229 & 1.175 & 1.174 \\
\hline $511+333$ & 1.308 & 1.342 & 1.343 & 1.399 & 1.278 & 1.263 & 1.323 \\
\hline 531 & 0.914 & 0.690 & 0.668 & 0.673 & 0.774 & 0.839 & 0.921 \\
\hline $600+442$ & 1.177 & 1.309 & 1.368 & 1.313 & 1.239 & 1.253 & 1.118 \\
\hline 620 & 0.923 & 0.943 & 0.918 & 1.026 & 0.950 & 0.864 & 0.900 \\
\hline 533 & 1.386 & 1.549 & 1.537 & 1.477 & 1.554 & 1,472 & 1.406 \\
\hline 622 & 0.844 & 1.035 & 0.946 & 0.896 & 0.842 & 0.891 & 0.828 \\
\hline 642 & 1.094 & 0.982 & 0.970 & 0.890 & 0.921 & 0.946 & 1.040 \\
\hline $731+553$ & 0.980 & 1.069 & 1.045 & 1.093 & 1.078 & 1.090 & 0.994 \\
\hline
\end{tabular}

with $g_{400}=1.51$. The most unfavorable orientation is [110] with $g_{220}=0.224$ since [110] may be perpendicular to [111] or $[100]$ for some of their variants.

By comparing $g_{h k l}$ calculated from the 16th order coefficients with the experimental results given in Table IV, one finds a $25.4 \%$ intensity reduction for (111) and $9.7 \%$ for (200). Alternatively, comparing the experimental results taking $g_{111}=g_{222}$, and $g_{200}=g_{400}$, one finds a similar intensity reduction, i.e., $25.7 \%$ for (111) and $9.3 \%$ for (200). If this was assumed to be due to primary extinction, by using Eqs. (2a)-(2c) and the parameters for the $\mathrm{Cu}-\mathrm{Be}$ alloy with $V_{c}^{2}=45.61 \AA^{3}$ and $g=0$. Then for Mo $K_{\alpha}$ radiation, one obtains $Q_{111}=0.238 \mathrm{~cm}^{-1}$ and $Q_{200}=0.180 \mathrm{~cm}^{-1}$, and $q_{111}=0.746$ and $q_{200}=0.903$ which are taken for $E_{111}$ and $E_{200}$ in Eq. (1a). With $t=0.44 \mu \mathrm{m}$, one finds that extinction reduces the (111) by 0.80 , the (200) by 0.85 , and (220) by 0.95 . In each case, the departure from the experimental result due to primary extinction is within the experimental error.

For the reduced intensity, the thermal term was calculated according to Eq. (11.77) and the Debye theory given in Ref. 5 with $\theta=315 \mathrm{~K}$. This gives a root mean square projected displacement of $0.085 \mathrm{~A}$. The static displacement is even smaller according to Eq. (3) i.e., $\left\langle\mu_{s}^{2}\right\rangle^{1 / 2}=0.027 \mathrm{~A}$ using data found in Ref. 8. Note the rms displacement is obtained from

$$
2 M_{i}=16 \pi^{2}\left\langle\mu_{i}^{2}\right\rangle^{1 / 2}\left(\frac{\sin \theta}{\lambda}\right)^{2}
$$

with $\mu_{i}$ projected along the normal to the diffracting planes $(h k l)$. These values give $k$ values that scatter about a constant value and are shown in Fig. 3. Since no systematic increase or decrease is observed with $(\sin \theta / \lambda)^{2}$ the sum of the attenuation factors, i.e., $M_{T}+M_{S}$, are in agreement with Debye and Krivoglaz theories. If this were not constant, this sum of thermal and static terms would have to be modified.

The normalized orientation function $\bar{w}(\Omega, \psi)$ in terms of 16th order coefficients are plotted in Fig. 4. These show that the orientation function is peaked at 2.32 for $(\Omega, \psi)$ $=\left(55^{\circ}, 45^{\circ}\right)$ and 1.51 for $\left(90^{\circ}, 0^{\circ}\right),\left(90^{\circ}, 90^{\circ}\right)$ and $\left(0^{\circ}, 0^{\circ} \sim 90^{\circ}\right)$ relative to a surface plane at $\bar{w}=1$, for the random powder. The minimum value of $\bar{w}(\Omega, \psi)$, located at $\left(45^{\circ}, 0^{\circ}\right)$, $\left(45^{\circ}, 90^{\circ}\right)$ and $\left(90^{\circ}, 45^{\circ}\right)$, is 0.224 and is 4 to 5 times smaller than the value for a random sample. Cubic symmetry is seen

TABLE V. Instrumental window factor for symmetric setup $(\chi=0)$.

\begin{tabular}{ccccccccc}
\hline \hline$\Delta_{X}$ & $\cos \left(\Delta_{X}\right)$ & $D_{4}$ & $D_{6}$ & $D_{8}$ & $D_{10}$ & $D_{12}$ & $D_{14}$ & $D_{16}$ \\
\hline 0.0 & 1.0000 & 1.0000 & 1.0000 & 1.0000 & 1.0000 & 1.0000 & 1.0000 & 1.0000 \\
0.5 & 1.0000 & 0.9998 & 0.9996 & 0.9993 & 0.9990 & 0.9985 & 0.9980 & 0.9974 \\
1.0 & 0.9998 & 0.9992 & 0.9984 & 0.9973 & 0.9958 & 0.9941 & 0.9920 & 0.9897 \\
1.5 & 0.9997 & 0.9983 & 0.9964 & 0.9938 & 0.9906 & 0.9867 & 0.9821 & 0.9769 \\
2.0 & 0.9994 & 0.9970 & 0.9936 & 0.9891 & 0.9833 & 0.9764 & 0.9684 & 0.9591 \\
2.5 & 0.9990 & 0.9952 & 0.9900 & 0.9830 & 0.9740 & 0.9633 & 0.9508 & 0.9367 \\
3.0 & 0.9986 & 0.9932 & 0.9857 & 0.9755 & 0.9628 & 0.9475 & 0.9297 & 0.9096 \\
3.5 & 0.9981 & 0.9907 & 0.9805 & 0.9668 & 0.9496 & 0.9290 & 0.9052 & 0.8784 \\
4.0 & 0.9976 & 0.9879 & 0.9746 & 0.9568 & 0.9345 & 0.9079 & 0.8774 & 0.8432 \\
4.5 & 0.9969 & 0.9847 & 0.9680 & 0.9455 & 0.9175 & 0.8844 & 0.8466 & 0.8044 \\
5.0 & 0.9962 & 0.9811 & 0.9605 & 0.9330 & 0.8989 & 0.8587 & 0.8130 & 0.7625 \\
\hline \hline
\end{tabular}




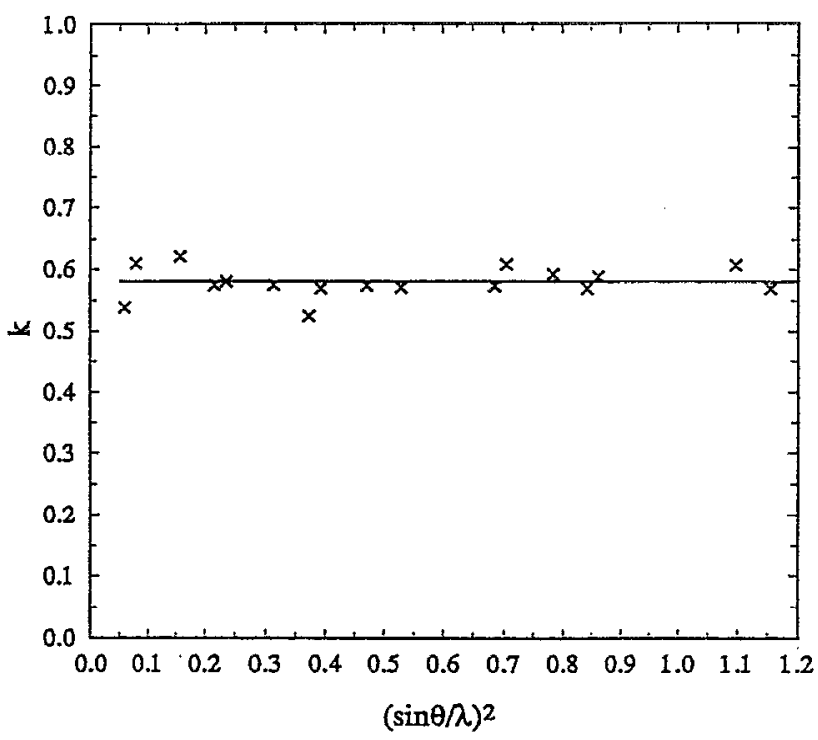

FIG. 3. Independence of $k$ vs $(\sin \theta / \lambda)^{2}$ after thermal diffuse, static diffuse and extinction correction.

about the $\psi=45^{\circ}$ axis. At $\Omega=0$, the fiber axis $z$ coincides with the reciprocal axis, $c^{*}$, making $\bar{w}(\Omega, \psi)$ independent of $\psi$. Therefore, the constant value of 1.51 at $\Omega=0$ for all $\psi$ anglès is to be expected.

Thus far, the data used to construct the orientation function can be obtained with a conventional diffractometer giving data at $\chi=0$. In order to obtain a more convincing check with experimental data, $\omega$ - rocking curves (or $\chi$ scans) were measured over a limited range. These are shown as points in Fig. 5 for (111), (200), and (220) pole densities. Having obtained a consistant set of coefficients describing the orientation function, one can generate any pole density function using Eq. (7b). Three are shown in Fig. 5 as dashed and as solid lines.

The true pole density plots were mathematically "smeared" with a correction due to the finite size of the "instrumental window." This window is determined from the

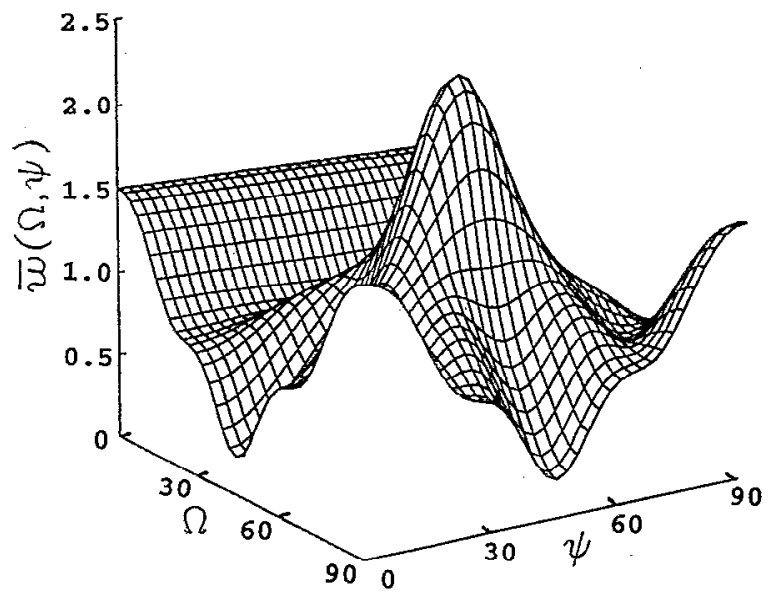

FIG. 4. Normalized orientation function $\bar{\omega}(\Omega, \psi)$ calculated from 16 th order.

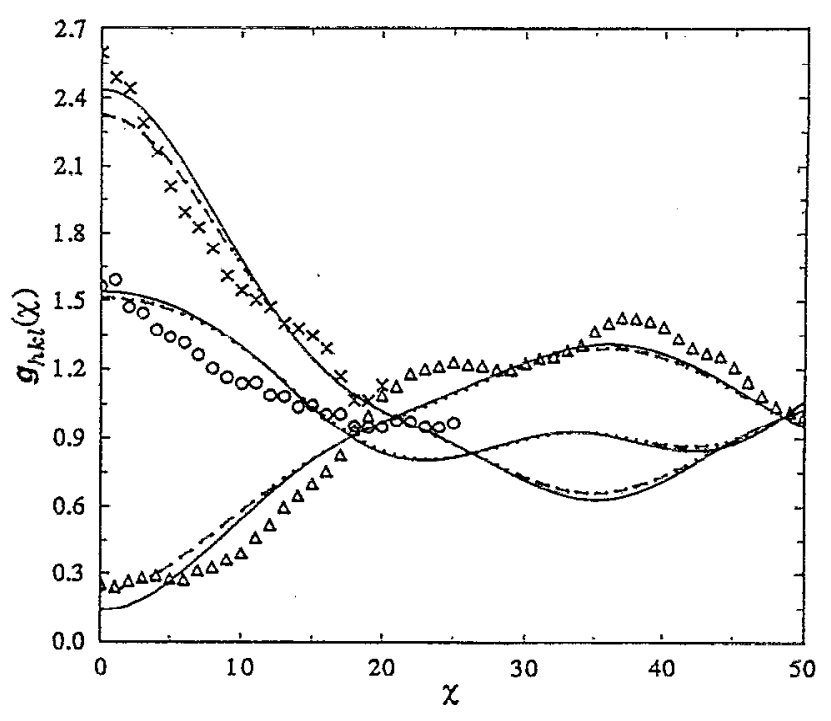

FIG. 5. Comparison of the pole density functions with experimental points $x-(111), o-(200),(220)-\Delta$ with $g_{h k l}(\chi)$ calculated from Eq. (6a) using 16 orders. The solid line includes a window correction while the dashed does not.

size of the x-ray focal spot, the receiving slit, and the amount of sample oscillation. It is treated in detail in the Appendix. The modification produced by this instrumental correction may be seen by comparing the dashed and solid curves of Fig. 5. The effect of the window correction is small in this example; however, it introduces an observable effect at $\chi=0$ for the (111) and (220). Pole figure smearing is expected to be larger for those diffractometers that are not of the focusing type when the texture is intermediate.

\section{B. Discussion}

Analytical procedures are presented that enable polycrystalline materials with a texture to be examined with the same detail as ideal powder samples. The added difficulty of doing a fundamental quantitative orientation analysis of the results offers the extra bonus of determining the fiber texture of the sample in addition to other parameters which are of interest by themselves. Thus far, the approach has been limited to cubic materials. When applied to a conventional parafocusing diffractometer, the approach remains simple if the texture does not show strong intensity variations with sample orientation; otherwise, a large number of independent Bragg peaks will be required for consistent results. Since many commerically available samples have textures that range from weak to intermediate, this is not a severe restriction on the approach.

Integrated intensity data for 17 diffraction peaks at $\chi=0$ provided the necessary input to determine the intermediate fiber texture for the extruded $\mathrm{Cu}-\mathrm{Be}-\mathrm{Co}$ alloy examined, as well as, the secondary parameters. The analysis is simplified with a tabulation of polynomial coefficients for a conventional symmetrically aligned diffractometer $(\chi=\omega=0)$ or one with asymmetric alignment $(\chi=\omega \neq 0)$ produced by an independent rotation of the sample. If the sample does not inher- 
ently contain a fiber texture, then spinning about its normal gives the average orientation function about this axis rather than the true orientation function which contains an additional angular dependence.

The overall approach requires that the intensity data be internally consistent with a single orientation function, the cubic symmetry of the sample, extinction influencing the strong low order peaks, and attenuation factors resulting from both thermal and static atomic displacements. Departures from theoretically generated intensities and reduced experimental intensities are due largely to statistical variations associated with the grain size.

The $\mathrm{x}$-ray optics with a conventional parafocusing diffractometer introduced only a small degree of smearing of the pole figure data because the texture is of intermediate sharpness. Some caution is advised, even with an intermediate texture, if data are collected using a conventional pole figure instrument with a point source, wide integrating slits, and no correction is made.

It was found that the displacement terms in a quenched Cu-11.67 at \% Be-0.23 at \% Co alloy can be described at room temperature by thermal and static displacements of $\left\langle\mu_{t}^{2}\right\rangle^{1 / 2}=0.085 \AA$ and $\left\langle\mu_{s}^{2}\right\rangle^{1 / 2}=0.027 \AA$. The latter could result from clusters containing an average of $1.7 \mathrm{Be}$ atoms. This is somewhat smaller than the cluster sizes determined by Koo et al. ${ }^{11}$ for an as quenched sample. However, their sample did not contain Co which reduces the rate at which clustering or aging takes place by about a factor of 5 . For the as quenched sample, the static contribution is dominated by thermal displacements. In a later work, we show that much larger clusters are formed in the same alloy during low temperature aging. Measurements of primary extinction suggest that the as-quenched subgrain size is approximately $0.44 \mu \mathrm{m}$ after annealing at $780^{\circ} \mathrm{C}$ for $15 \mathrm{~min}$. A similar sample annealed at $810^{\circ} \mathrm{C}$ for 20 min resulted in a subgrain size of $0.66 \mu^{12}$ which was also obtained from a primary extinction analysis. This is in agreement with the present determination if one considers the higher temperature and longer annealing time used in sample preparation for the latter determination. Also, when the latter sample was subsequently aged at 200 and $315^{\circ} \mathrm{C}$ to form GP zones, this practically elliminated extinction effects as a result of the matrix deformation associated with aging.

\section{ACKNOWLEDGMENTS}

Funding for this research was made available by $\mathrm{Na}-$ tional Science Foundation Grant No. DMR-8818013. We are grateful to Dr. Amitava Guha (Brush Wellman Inc., Cleveland, $\mathrm{OH}$ ) for providing samples and his preliminary discussions that provided a very useful background for this research.

\section{APPENDIX}

\section{Instrumental window correction}

In the analysis of texture, it is commonly assumed that the diffracted intensities are measured exactly at $\chi$. This is not true because the instrumental window of the diffractometer will always give a range of $\chi$ angles in the vicinity of the central angle $\chi_{0}$. Consequently, the measured pole density is an average value of $g_{h k l}(\chi)$ in the range between some lower limit $\chi_{1}$ and an upper limit $\chi_{2}$

$$
g_{h k l}^{m}\left(\chi_{0}\right)=\frac{\int_{\chi_{1}}^{\chi_{2}} W(\chi) \cdot g_{h k l}^{t}(\chi) \cdot \sin (\chi) d \chi}{\int_{\chi_{1}}^{\chi_{2}} W(\chi) \cdot \sin (\chi) d \chi} .
$$

$g_{h k l}^{m}\left(\chi_{0}\right)$ is the measured value at $\chi_{0}$ and $g_{h k l}^{t}(\chi)$ is the true value of the pole density function. $W(\chi)$ is the weighting factor of the instrumental window in the range of $\chi_{1}$ to $\chi_{2}$. If the average expressed in Eq. (A1) is compared with Eq. (6a), we obtain

$$
g_{h k l}^{m}\left(\chi_{0}\right)=\sum_{n=0}^{\infty} G_{n}^{h k l} \sqrt{\frac{2 n+1}{2}} P_{n}\left(\cos \chi_{0}\right) \cdot D_{n},
$$

where $D_{n}$ is the instrumental window factor and $P_{n}\left(\cos \chi_{0}\right)$ are unnormalized Legendre polynomials. The window factor is given by

$$
D_{n}=\frac{\int_{\chi_{1}}^{\chi_{2}} W(\chi) \cdot P_{n}(\cos \chi) d \cos (\chi)}{P_{n}\left(\cos \chi_{0}\right) \cdot \int_{\chi_{1}}^{X_{2}} W(\chi) d \cos (\chi)} .
$$

For simplicity, take $W(\chi)=1$ everywhere in the integration range. From Ref. 13

$$
\int P_{n}(t)=\frac{1}{2 n+1}\left[P_{n+1}(t)-P_{n-1}(t)\right]+C .
$$

Therefore,

$$
D_{n}=\frac{P_{n+1}\left(\cos \chi_{2}\right)-P_{n-1}\left(\cos \chi_{2}\right) \mp P_{n+1}\left(\cos \chi_{1}\right) \pm P_{n-1}\left(\cos \chi_{1}\right)}{(2 n+1) P_{n}\left(\cos \chi_{0}\right)\left[\left(\cos \chi_{2}-1\right) \mp\left(\cos \chi_{1}-1\right)\right]}
$$

where the upper sign applies to the case $\chi_{2}>\chi_{1} \geqslant 0$ and lower sign applies to the case $\chi_{2} \geqslant 0>\chi_{1}$. With symmetrical optics, $\chi_{0}=0, \chi_{1}=-\Delta \chi, \chi_{2}=\Delta \chi$, and $P_{n}\left(\cos \chi_{0}\right)=1, D_{n}$ is simplified to

$$
D_{n}=\frac{P_{n+1}(\cos \Delta \chi)-P_{n-1}(\cos \Delta \chi)}{(2 n+1) \cdot(\cos \Delta \chi-1)} .
$$

The angular window of the instrument is determined from the size of the focal spot, receiver slit, and sample oscillation angle. The range in $\chi$ for the symmetrical case may be approximated by

$$
\Delta \chi=\sqrt{\Delta \chi_{h}^{2}+\Delta \chi_{v}^{2}}
$$

where $\Delta \chi_{h}$ is the max of $\Delta \omega, \alpha,\left(W_{e} / 2 R\right)$ or $\left(W_{r} / 2 R\right)$ and $\Delta \chi_{v}$ is the max of $\beta,\left(L_{e} / 2 R\right)$ or $\left(L_{r} / 2 R\right)$ divided by $\sin \theta$. Here $R$ is the sample-to-receiver slit distance, $L_{e}$ is the length 
of focal spot, $L_{r}$ is the length of receiver slit, $\beta$ is the axial divergence of the Soller slit, $W_{e}$ is the width of focal spot, $W_{r}$ is the width of receiver slit, $\alpha$ is the divergence allowed by the entrance slit, and $\pm \Delta \omega$ is the sample oscillation angle.

Data were obtained by rotating $\omega$ rather than tilting in $\chi$. In this asymmetric geometry (at a tilt angle $\omega=\omega_{0}$ ), the above equations do not hold, since the diffractometer is not in the focusing condition. If $\Delta \omega$ is $\geqslant \alpha,\left(W_{e} / 2 R\right)$ and $\left(W_{r} / 2 R\right)$, the range of $\chi$ can be approximated by

$$
\begin{aligned}
& \chi_{1}=\omega_{0}-\Delta w, \\
& \chi_{2}=\sqrt{\left(\omega_{0}+\Delta \omega\right)^{2}+\left(\frac{L_{e}+L_{r}}{2 R \sin \theta}+\frac{\beta}{\sin \theta}\right)^{2}}, \\
& \chi_{0} \equiv \omega_{0} .
\end{aligned}
$$

$D_{n}$ can be determined by Eq. (A5).

The instrumental window factors $D_{n}$, for $0 \leqslant \Delta \chi \leqslant 5^{\circ}$ about $\chi_{0}=0$ are given in Table IV for up to the 16th order. $D_{n}$ depends on the window size $\Delta \chi$, and becomes more severe at higher orders. At an extreme of $\Delta \chi=5^{\circ}, D_{4}=0.981$, while $D_{16}=0.763$. This suggests that the instrumental window correction is negligible for weak to intermediate textures where high order coefficients become trivial. As the pole density data sharpen, this correction becomes more significant. To make the window correction, $D_{n}$ should be introduced into Eqs. (7a) and (7b), giving

$$
g_{h k l}=1+\sum_{n=1}^{N} \sum_{m=0}^{n} D_{n} C_{h k l}^{n m} A_{n m}
$$

or

$$
g_{i}=1+\sum_{j=1}^{q} C_{i j}^{\prime} A_{j}(i=1,2, \ldots . .,),
$$

where $C_{i j}^{\prime}$ represents $D_{n} C_{h k l}^{n m}$. Equation (7b) is still valid with the instrumental window included provided that $C_{i j}^{\prime}$ is used in place of $C_{i j}$. After the coefficients $A_{n m}$ are fitted to the data with the window included, the true unsmeared pole density function can be obtained from Eq. (7b), i.e., without the inclusion of $D_{n}$.

${ }^{1}$ R. J. Roe and W. R. Krigbaum, J. Chem. Phys. 40, 2608 (1964).

${ }^{2}$ H. J. Bunge, Texture Analysis in Materials Science (Butterworths, Boston, MA, 1982) (translation by P. R. Morris).

${ }^{3}$ S. Rao and C. R. Houska, J. Appl. Phys. 54, 1872 (1983).

${ }^{4}$ L. H. Schwartz and J. B. Cohen, Diffraction from Materials (Springer, New York, 1987).

${ }^{5}$ B. E. Warren, $X$-Ray Diffraction (Addison-Wesley, Reading, MA, 1969), p. 190.

${ }^{6} \mathrm{M}$. A. Krivoglaz, Theory of X-Ray and Thermal-Neutron Scattering by Real Crystals (Plenum, New York, 1969), p. 234.

${ }^{7}$ A. Guinier, X-Ray Diffraction in Crystals, Imperfect Crystals, and Amorphous Bodies (W. H. Freeman, San Francisco, CA, 1963), p. 113.

${ }^{8}$ H. W. King, J. Mat. Sci. 1, 79 (1966).

${ }^{9}$ R. J. Roe, J. Appl. Phys. 37, 2069 (1966).

${ }^{10} \mathrm{~S}$. Rao, PhD dissertation, Dept. of Materials Engineering, Virginia Tech, 1984.

${ }^{11}$ Y. M. Koo, J. B. Cohen, S. M. Shapiro, and L. E. Tanner, Acta Metall. 36, 591 (1988).

${ }^{12}$ Ya. S. Umansky, V. I. Yelyutina, A. S. Kagan, and L. Kh. Pivovarov, Kristallografiya 2, 503 (1957).

${ }^{13}$ T. M. MacRobert, Spherical Harmonics, 3rd ed. (Pergamon, New York, 1967), pp. $79-112$. 